\section{PLANT PATHOLOGY: TEACHING AND RESEARCH*}

\author{
By Prof. W. BROWN, F.R.S.
}

$\mathrm{W}$ E are now, we hope, approaching the realization of peace and of that kind of atmosphere in which biological work, and more especially research, can hope to flourish once more. We have every reason for believing that the.next few years will see many changes-changes in our educational system at all levels, with resultant effects upon students who take up an applied biological profession, and changes in our agriculture which will lead to a different emphasis upon instruction and research in biological subjects. With these changes impending, it may therefore be opportune at this time to look back over the years of one's experience, and see how far the old system appeared to be satisfactory and where on the other hand it did not give the results which might have been expected.

As the scope of plant pathology varies somewhat from place to place, it will be well, first of all, to say a few words on this topic. My own experience has been substantially in the region of mycological plant pathology, that is, I am a plant pathologist in the narrower sense which is current in Great Britain, as for example in the calendars of universities and agricultural colleges. This narrower definition leads to difficulties in practice, as for example in delimiting the respective spheres of the plant pathologist and the agricultural entomologist in the study of insectborne diseases. It is much better, I think, to use the words 'plant pathology' in a wider sense, and to mean by it the study of the plant in disease, whatever the cause may happen to be.

On this topic of definition, I would venture to go even further towards generality. The word 'disease' has come to acquire rather a special meaning, and to most people implies more than mere 'unsatisfactoriness'. I would be prepared to stake a claim as follows. Wherever a plant (or a plant product) is unsatisfactory for any reason whatever and in any respect whatever, as for example when the yield has been smaller in quantity or less desirable in quality than might reasonably have been expected, there one sees a problem for the plant pathologist. No doubt it would be very easy to state problems which fall within this widest scope and which would sound curiously if labelled 'plant pathological'. Such matters appeal to me as details, and the important point is to recognize the unity of aim, which is to achieve more and more control over the plant in certain desired directions. The technicians employed must of necessity be various-entomologists, geneticists, mycologists, soil chemists and others-but they are all harnessed to the one major purpose, the production of more and better plants. I need scarcely add that this is the main interest of the public, who supply in the long run the funds which render our work possible.

I have put in my title the phrase: "Teaching and Research". The two are not easily separable, for there is general agreement that teaching will not long be very live if the research side is dead. The necessity. for research in a teaching institution, quite apart from the desirability of keeping the name of the place

- Substance of the presidential address given before the Association of Applied Biologists on February 23. upon the map, is all the more compelling if it is agreed that the object of teaching is not so much to inculcate a mass of facts which may soon be forgotten, as to instil methods of handling facts, and where possible of discovering new ones.

It would take too long to go into any detail as to what I think is the most desirable pre-graduation training for a man who intends to take up plant pathology as a career. The old system, or rather lack of system, under which we have been brought into the subject, can at least claim that it functioned in the best tradition of natural selection. But clearly one must set some limits to this sort of thing in the interests of efficiency and the saving of time. I would like to make some general observations under this heading.

First of all, what type of man, from the secondary schools, should we welcome as entrants into the biological field? Here I am giving my personal opinion, though I know that many of my colleagues are in agreement with me on this point. We must look here at the kind of training given in the schools.

There was a time, not so long ago, when botany was the only biological subject which found a place in the school curriculum. This applied only to a proportion of schools, and in these it was not on a level with the physical sciences. It was in general a subject for girls and for the weaker of the boys. In the ten years or so before the War there was a considerable change-over from botany to the composite subject biology. This movement was progressive and extended to schools which had not hitherto offered instruction in any biological subject. The expectation is that this development will continue after the War, so that we can look to a not-distant date when biology will take its place as a standard subject in schools, much on a level with physics or chemistry. Considered from the point of view of equipping the ordinary citizen for his life's work, this change has a great deal to recommend it, and I have not met anyone who seriously contests its merits. We are concerned here with the more limited question as to how it affects the equipment of students who go up to the universities bent upon a career in biology.

Frankly, I would say that we would rather that entrants into university biological departments did not have this preliminary biological training at school, and that for two reasons. One of these is probably temporary; the other, I fear, is permanent. As regards the first, we are still in the transition period, which may last for some time. For reasons which are sufficiently obvious, the standard of instruction at present reached varies very greatly from school to school, so that in the mixed batches who go up to the universities it is difficult to know where best we should begin. It is particularly with regard to laboratory instruction that we find the greatest unevenness, and obviously that is very important. No doubt this difficulty will lessen as times goes on. The second objection, of a more lasting nature, is this. Biological instruction in the schools has been developed at the expense of the physical sciences, and there is a grave risk that the biological student of the future will go up with an inferior grounding in these fundamental subjects. Once he has started on his biological work, he may never have the opportunity or the will-power to go back and make good the omission, and so the field of his usefulness may be permanently narrowed. Speaking broadly, one can say that there are some subjects of a kind that if one neglects them in one's 
youth, one does not easily pick them up later. Physics, and more especially mathematics, are such subjects.

Summarizing what I have just said, I would picture the desirable type of student somewhat as follows. It is immaterial whether he has had any biological instruction or not; but he should be well founded in the basal sciences, and also have a natural interest in plants or animals. A priori, I would rather that he were a countryman who preferred the country to the town, as he would be more likely to feel at home there and to have a broad country background.

The choice of training centre for such a student lies between the biological departments of a university, which nearly always is situated in a large town, and an agricultural college, which is usually in the country. Both have their merits and limitations.

Students at an agricultural college have the advantage that they are not brought up in a theoretical or academic atmosphere; they are in country surroundings and are in contact to some extent with the practical side of things. At the same time I am not prepared to allow that a few years' or even a lifetime's residence at an agricultural college necessarily imparts the true inwardness of the farming life. Many farmers draw a sharp distinction between what is done at an agricultural college and what they consider to be practicable on the average farm. From the technical point of view the great objection to the ordinary agricultural college as a place for the training of specialists in the plant industry is the meagreness of instruction in the basal sciences. A sound and comprehensive course in the biological sciences seems to me to be essential, and that is what the agricultural colleges are not at present in a position to give. Of course, one must allow for exceptional cases, and I know personally some excellent research workers who have graduated by this channel. Nevertheless I consider them to be exceptional cases.

The strength of the university department is the weakness of the agricultural college, and vice versa. I need not dilate upon the social advantages which accompany the university life; but shall confine myself to the technical. It is of the greatest value to a student, in his undergraduate years and perhaps more so during a period of postgraduate research, to be able to rub shoulders with workers in other scientific subjects or in university subjects generally, with consequent broadening of his outlook. There are also the special advantages of access to advanced. courses on topics related to his main study, for example, courses on physical chemistry, spectroscopy, and so on. These advantages are obvious; the disadvantages require fuller treatment.

The question has been seriously asked: Is the rarefied atmosphere of a university biological department a suitable training ground for a man who is going to specialize in problems of plant culture? Prof. W. B. Brierley, in his presidential address of some ten years ago to the Association of Applied Biologists, discussed this point in a very lively manner and had much to say about the academic man sicklied o'er with the academic cast of thought. With much of what he said I find that I am in hearty agreement, though I am optimistic enough to believe that there has been an improvement in the interval. I do not think that you will find so many of the academic 'die-hards' flourishing in university. circles as you would have found twenty years ago. It is also true that courses in botany and zoology. are brought into line with current interests and activities, by the introduction of new and the scrapping of old material, though it may well be that the process of modernization is not going on fast enough.

Speaking in particular of botanical courses, I should say that the greatest weakness is the lack of intimate contact with growing plants. By intimate contact I do not mean merely looking at living plants ; I mean actually handling them and growing them. It does not follow from this that I would like to train botanical students as gardeners or farmers, but I do hold that a training which deals largely with preserved plants and which is centred in a laboratory is lop-sided. Every effort should be made to bring the young student, from the earliest stage of his career, into contact with the practical growing of plants. This is desirable for all botanical students, but more especially for those who intend to take up an applied side such as plant pathology. Practical contacts can be developed by arranging for students to work at research institutes or on farms during vacation periods, and it is clearly desirable that the facilities in university departments for experimentation in the field or glasshouse should be extended. Granted that such facilities are fortheoming, I feel sure that the proper place for the pregraduate training of the plant specialist, including the plant pathologist, will continue to be the university biological department.

The field of research in plant pathology which is appropriate for a university staff (as contrasted with that of a research institute) is no doubt fairly wide; but there are some obvious limitations, even when the facilities of laboratory and experimental grounds are all that could be desired. The subject chosen should be one which could be expected to yield a result within a period of about two years, even though the primary object is not to obtain publishable results as such but to give training in research methods. Two years is a short time for outdoor research, where the kind of work possible is often dictated by the season, and especially when, as often happens, the first season must be largely given over to exploratory work and to the assembling of suitable material. A useful plan in my experience has been to assign to each student two problems, one involving laboratory work and often academic in outlook which could be followed up at any time of the year, and the other an outdoor one of a seasonal nature. This has a certain insurance value from the point of view of results, and is particularly useful in helping to tide the student over the earlier slack, and sometimes bleak, phases of the work.

Teaching and research of the kind which I have been describing, helped by my natural inclination in these matters, have had the effect of bringing me into somewhat close contact with the practical grower and his problems. It happens also that I come of farming stock and am personally acquainted with many farmers and their ways of looking at things. I may therefore be allowed to put down some of my reflexions on the functioning of the research machine, and these will fittingly conclude this address. As there will be some criticisms, I wish to make it clear that henceforth I shall be speaking of plant pathology in the narrow sense where my own experience lies and where I am surer of my ground.

First of all there is the much-debated question of the relative importance of academic and applied research, not merely from the point of view of research as such, but also of research as leading to results of practical value. It has always seemed to 
me that, in discussions on this topic, much confusion of thought has arisen from a more or less unconscious misuse of the rather numerous names which have been applied in this connexion. If we say that there are, broadly speaking, two types of research, the theoretical which aims at understanding phenomena without reference to any practical application of its findings, and the practical which aims at a definite practical result, there will, I think, be general agreement that both are important and desirable, and in many cases there will be found to be an interdependence between the two. Unfortunately it has been customary to apply a superfluity of names to each type of research, and the use of some of these names has led to misconceptions and, in my opinion, to some misdirection of research. Thus, the theoretical type is known as 'academic', 'fundamental', 'basal' or 'long-term', and the practical as 'applied', 'ad hoc' or 'short-term'. I would like to comment upon some of these terms.

If by 'fundamental' is merely meant 'theoretical' as roughly defined above, I have no objections to it. There is, however, a strong tendency to read a further meaning into the word. Just as a house cannot be built unless its foundations or fundaments are properly laid, so, it is argued, must the practical work follow and rest upon the earlier fundamental investigation. One hears it freely stated that from such fundamental work results of practical importance drop out from time to time, and all that remains for the practical man is to recognize them as such or to seize upon certain principles, proceeding from which he can readily arrive at practical results. Now I am well aware that there is no great difficulty in furnshing examples in support of such views, more notably in certain physical arts-such as radiolocation-in which the practice has been built up upon results which a few years ago were of theoretical interest only. The relation of the biological sciences to agriculture is, however, somewhat different, as I need scarcely enlarge upon. Most of the results of practical value have been obtained by the growers themselves-sometimes by accident-and in such cases the function of agricultural science has been in the main to explain the results, to point out their limitations and sometimes to indicate improvements. It is well to recognize the fact that results of the very greatest practical importance have been obtained in connexion with problems the theoretical basis of which is quite unknown to this day. For example, it has been possible to breed potatoes which to all intents and purposes solve completely the problem of wart disease, and yet we are still quite ignorant of the theoretical basis of this very valuable result. Similar examples will no doubt occur to everyone.

It must not be thought that, in speaking as I have done, I am disparaging the theoretical type of research. I should be roundly accusing myself if I did so. It is obvious that theoretical research in biology generally, and in plant pathology in particular, has the same status as it has in any other science or form of learning - that it is worthy of being carried on, and carried on vigorously, on its own account. It has cultural value and adds its quota to the progress of civilization. It tends to fill in the general scientific picture, and from the point of view of the research worker himself it helps in many cases, if I may use the phrase, to keep his soul alive. It is, however, idle to maintain that practical results cannot be obtained without theoretical research, and it has always appeared to me that it is undignified, to use a mild term, to put forward as a justification of theoretical research the possibility that results of practical value may arise from it by accident. No doubt if pursued long enough and with sufficient insight, it may point the way to a solution of all our practical problems, but that date is not yet. The prectical method, I feel, must still be to tackle the practical problem with the means at one's disposal, and in so far as difficulties arise to refer these back for closer investigation in the hope that new insight may be obtained. In other words, the practical method should be that of direct attack, with theoretical research harnessed to it for purposes of guidance and further development.

The description 'long-term' as applied to theoretical research is appropriate. I should be happy to see it replaced by 'everlasting', for the more successful a piece of theoretical research proves to be, the more does it suggest further problems. On the other hand, I heartily deplore the tendency to apply the word 'short-term' to applied research. I have no objection to the phrase ' $a d$ hoc', in so far as it means that there is a definite objective; but it is unfortunate that the adjective 'definite' is so often paraphrased to 'limited' and so to 'short-term', which in many cases has come to mean 'two years or thereabouts'-the normal duration of a research scholarship. The definiteness of an objective has no obvious relation to the time required to reach it, and in biological research, especially when its results are sharply conditioned by seasonal factors, it is surprising how long a time is necessary for the proper working out of even a simple problem. If, for example, it be the matter of the use of a fungicide, even when the best form of fungicide and the mode of its application have been settled, a great deal of work remains in testing its efficiency in various situations and over the variety of conditions which arise in a succession of seasons. I do not believe that the time-consuming nature of much applied biological research has been properly appreciated by those responsible for its organization, and to this more than to anything else is to be ascribed the fact that much research has failed to reach the farmer in a form usable by him. I shall refer to this point again.

The inadequate provision for the ' $a d$ hoc' type of research is illustrated in another way. Fundamental research, from its long-term nature, and because it gives no promise of early results, is not the kind of thing which the cultivator could be expected to subsidize. It must rely therefore upon official support, and examples of reasonable support for it are familiar to all. On the other hand, it has often been expected that for work of the ' $a d$ hoc' type the industry concerned should pay a substantial share of the cost. This would seem on the face of it to be a reasonable arrangement, and it has in fact functioned, but there are inherent difficulties. Perhaps I can illustrate this point by an experience of my own. When an investigation of the carnation wilt problem had been in progress for two years, support for its continuation was sought from the carnation growers. Very naturally the lead was taken by certain members whose nurseries were at the time being seriously ravaged by the disease. Others, however, were as yet untouched and therefore not so interested. It was freely hinted that the trouble might not be a bad thing in itself, as tending to prevent overproduction and to weed out the inferior growers. Later, it may be added, the disease spread to other nurseries so that some growers had to change their 
opinions. One grower said that he knew of a cure, and another that he did not propose to subscribe, but that he would pay for the cure when found. It so happened that there was present a man of very great importance in the industry who took the line that though his firm was not as yet seriously troubled by the disease, the industry as a whole was in danger, and it was highly desirable that he should have as much information as possible in advance of its coming. So the scheme came to be supported. I quote this example, not so much to illustrate how vexatious and chancy the initiation of research in this way may be, but to stress the fact that the financial interests of the growers of any one commodity are not necessarily identical.

Perhaps I should indicate here what, in my view, is the solution of this problem. The effect of the successful application of research to a problem of plant production is that the yield of produce of a certain quality is increased. This might lead either to a reduction in cost to the public, that is, to a raising of the standard of living, or to a reduction in the amount of land necessary for growing the required amount of produce, an economy which is obviously desirable in a small country such as Great Britain. In either event the advantage is national, and therefore the responsibility for initiating and supporting such ad hoc work should rest upon national funds, even though some growers may benefit, temporarily or permanently, from the results obtained.

I am hopeful that in the near future we shall see some improvements in the organization of plant pathological research. Hitherto, except for a few cultivations such as fruit, applied research has been of a very occasional and scattered nature. There has been little co-ordination, and, so far as I can see, a lack of purposefulness in following up the 'worthwhile' problems to a stage where the results could be confidently brought to the notice of cultivators. It is the function of no one in particular to collate the work which has been done on a particular problem and to devise steps for carrying it forward to a practical solution. When such a survey is made, I am afraid that it will reveal a scarcity of practical methods of proved value, for the conditions under which much research has been done have been such as to limit its scope to purely mycological phases. I am not being facetious when I suggest that this state of affairs is reflected in the abundance of control measures which figure in plant pathological textbooks. I am quite certain that many of the methods put forward are of doubtful practicability or usefulness, and that very few have been worked out in a manner which would convince a practical man.

The number of problems awaiting a practical solution is so great that a rigorous selection would be necessary to allow of adequate attention being paid to those deemed most important. What we require is a 'priority list' of problems, and when such a list is drawn up, I hope that the views of research workers and growers will be sought more freely than has been the case in the past.

The practical aim of plant pathological research is not to study plant disease as such, but to point the way to growing good plants in spite of a liability to disease. It seems obvious, therefore, that research should be organized on a crop basis. There must be research institutes for the main crop plants, with small and possibly movable field stations where the more local problems can be investigated on the spot.
We have moved some distance in this direction and I think there will be a fuller development in the not distant future.

As regards the connecting link between research worker and grower, I speak mainly as a spectator. There is just one point which I wish to make in this connexion.

It has often been stated that research workers have in their possession a mass of information which the growers do not put to practical use, either because it is not brought to their notice at all, or if so, not in a form which they can understand. The blame for this tends to be laid upon the advisory organization. I would like to record what has been a frequent experience of mine when visiting nurseries in the Reading Province in company of the advisory mycologist. The object of the visit might relate to one particular problem, but by the time we had gone half-way round the nursery a dozen other queries had been put. There was obviously a multitude of problems, to many of which it was impossible to give a definite answer. Sometimes one could say that "Somebody in America had described a thing rather similar". The plain fact is that there are many growers' problems-of all kinds and dimen- sions-to which no clear answer can be given, because the necessary research work has not been done. This is a point which I have already stressed. Doubtless the advisory services could be made to function more efficiently-for example, by arranging better facilities for the demonstration of approved methods of culture - but I feel sure that, in the plant pathological field at least, the main lack is in the paucity of appropriate research, and not in the inability of the advisory services to convey such useful information as exists.

To sum up-there is no doubt as to the desirability and feasibility of tightening up our methods of training students for applied biological work, both in their undergraduate years and in their training as research workers; but I feel certain that the greatest requirement is a well-directed concentration upon the problems which the practical grower is prepared to put before the scientific worker. There are many such, and if, as appears to be very likely, there is to be a continued intensification of agriculture in Great Britain, there will be many more in the future. There is no doubt, therefore, as to the magnitude of the field, and it is a responsibility devolving upon us, as scientific workers, to show that the practical problems of plant cultivation can be solved by the methodical application of the scientific method.

\section{PSYCHOLOGICAL IMPLICATIONS OF THE CULTURE-PATTERN THEORY}

$\Delta \mathrm{T}$ the meeting of the British Psychological A Socioty during April 5-10 in Exeter, a symposium was held on "Psychological Implications of the Culture.Pattern Theory". The speakers were Lieut.-Colonel R. F. Barbour, Dr. J. C. Flugel and Prof. T. H. Pear.

Prof. Pear opened by remarking that Ruth Benedict's "Patterms of Culture" was now ten years old; it had affected some writers upon psychology who use the concept, but few attempts have been made to appraise the numerous psychological implications in the writings of Benedict, Margaret Mead, Bateson, Maslow and Clyde Kluckhohn in the 